\title{
Are ethnic differences in insulin sensitivity explained by variation in carbohydrate intake?
}

Received: 4 October 2004 / Accepted: 14 February 2005 / Published online: 28 April 2005

(C) Springer-Verlag 2005

\begin{abstract}
Aims/hypothesis: Minority populations are disproportionately affected by diabetes. This health disparity may be due to less healthy diets and/or heritable factors in minority populations. These factors must be assessed concurrently to better appreciate their contribution to insulin sensitivity. Methods: We analysed overweight, healthy adults using the National Health and Nutrition Examination Survey 1999-2000. Means for dietary intake variables and insulin sensitivity were calculated by ethnicity. Linear regressions were performed to evaluate the association between ethnicity, dietary variables, dietary glycaemic index and insulin sensitivity. Fasting insulin was used to characterise insulin sensitivity. Results: Non-Hispanic whites have higher energy and fat intake, while Hispanics have higher carbohydrate intake and AfricanAmericans have lower fibre intake. In unadjusted analyses both Hispanics and African-Americans have lower insulin sensitivity, but only Hispanics are more likely to have lower insulin sensitivity after controlling for dietary variables and BMI. Conclusions/interpretation: Ethnic differences in insulin sensitivity remain after controlling for dietary differences and other factors, suggesting that inherent metabolic differences exist. Further studies are needed to define inherent metabolic factors, as well as other nondietary factors that affect insulin sensitivity.
\end{abstract}

Keywords Carbohydrate intake - Glycaemic index • Health disparities · Insulin sensitivity · Minority populations

\footnotetext{
V. A. Diaz $(\bowtie) \cdot$ A. G. Mainous III · R. J. Koopman ·

M. E. Geesey

Department of Family Medicine,

Medical University of South Carolina, 295 Calhoun Street,

PO Box 250192 Charleston, SC, 29425, USA

e-mail: diazva@musc.edu

Tel.: +1-843-7927241

Fax: +1-843-7923598
}

\begin{abstract}
Abbreviations NCHS: National Center for Health Statistics - NHANES: National Health and Nutrition Examination Survey
\end{abstract}

\section{Introduction}

Type 2 diabetes mellitus is a common chronic disease that is characterised by insulin resistance. People with diabetes are at increased risk of several serious complications, including retinopathy, renal disease and cardiovascular disease [1-7]. Minority populations, such as Hispanics and African-Americans, are disproportionately affected by diabetes $[8,9]$. The cause of this disparity is probably multifactorial, based on differences in access to care, cultural factors and health beliefs, as well as genetics.

Certain diets that have a specific intake of dietary factors such as fat, cholesterol and fibre are helpful in controlling diabetes and its complications $[10,11]$. Thus, cultural differences in dietary intake may affect the prevalence of diabetes in minorities, since in general, minorities have less healthy diets. For example, several studies have suggested that Hispanic-Americans have lower vitamin intake than their non-Hispanic white counterparts $[12,13]$. An assessment of adults in Los Angeles and Hawaii showed Hispanics have a higher mean daily energy intake than nonHispanic whites, as well as higher fat intake [14]. Studies evaluating dietary intake in African-Americans are not consistent, but several studies show this group has less healthy diets than non-Hispanic whites [12, 14-19].

Inherent metabolic differences by ethnicity may also affect the risk of developing diabetes. Several studies suggest that minorities are more prone to decreased insulin sensitivity even after controlling for other risk factors. For instance, African-Americans without diabetes are more likely to have decreased insulin sensitivity than non-Hispanic whites, even after controlling for obesity and body fat distribution [20]. Similarly, insulin and C-peptide levels have been shown to be higher in Hispanics than in nonHispanic whites, again even after adjustment for obesity and body fat distribution [21]. However, these studies 
do not adjust for the presence of dietary differences by ethnicity.

Diet and heritable factors need to be assessed concurrently to better appreciate their contribution to insulin sensitivity. Much of the ethnic disparity seen in the prevalence and severity of diabetes may in fact be due to dietary differences based on cultural factors. This study uses ethnicity as a broad marker for known and unknown heritable factors, evaluating its association with insulin sensitivity once diet is taken into account. This process allows us to evaluate whether ethnicity and the associated heritable factors are still important determinants of insulin sensitivity once dietary differences are considered. We studied overweight adults without diagnosed diabetes or cardiovascular disease in order to evaluate a population at higher risk that has not received disease-specific medical intervention, thereby minimising disparity due to differing health care utilisation. This study was carried out in accordance with the Declaration of Helsinki as revised in 2000.

\section{Materials and methods}

Survey description Data from the 1999-2000 National Health and Nutrition Examination Survey (NHANES) were analysed. The NHANES is a product of the National Center for Health Statistics (NCHS). It is a continuous, annual survey involving participants from a nationally representative sample of non-institutionalised residents of the United States. The participants gave informed consent for their inclusion in this survey. Minorities were oversampled to ensure adequate numbers for analysis, which allows for more accurate population estimates. The NHANES includes a detailed household interview and physical examination, plus laboratory information obtained through mobile examination centres.

Samples are weighted so they are representative of the US population. Sampling weights are calculated taking into account unequal probabilities of selection due to sample design, non-response and planned oversampling, and then matched to known population control totals to be representative of the US population. This allows us to make population estimates for the United States.

Sample Overweight adults (BMI $>25,>18$ years old) were included in this sample. Respondents with a self-reported history of stroke, myocardial infarction, hypertension, high cholesterol, heart failure, angina and/or coronary heart disease were excluded. Respondents with diabetes were also excluded, providing us with a sample of normoglycaemic individuals. Respondents with dietary data that were deemed unreliable by the NCHS were also excluded.

Demographic data We categorised individuals by ethnicity based on a self-report as non-Hispanic white, AfricanAmerican or Hispanic. Personal history of disease was also based on self-reports. BMI was calculated from measured weight and height (weight in $\mathrm{kg} /$ height in $\mathrm{m}^{2}$ ). A BMI $>25$ was classified as overweight, consistent with
1998 National Heart, Lung and Blood Institute guidelines [22].

Outcome variable: insulin sensitivity Insulin sensitivity was measured using fasting insulin. Fasting insulin has been validated as the most appropriate single laboratory measure to describe insulin sensitivity in normoglycaemic individuals by comparing it with the euglycaemic insulin clamp method [23, 24]. Fasting insulin was analysed as a continuous variable.

Dietary variables The NHANES quantifies dietary intake in the $24 \mathrm{~h}$ prior to the interview via dietary recall interviews that were conducted in person by trained dietary interviewers fluent in Spanish and English. If necessary, translators were available for respondents who speak other languages. A 'multiple pass' method was used to obtain dietary information. This entailed obtaining an initial list of foods consumed, after which respondents were asked about the time and place of consumption. A list of frequently forgotten foods was then displayed, and a complete description of the foods eaten obtained. Finally, the foods were reviewed in chronological order with amendments made as appropriate. A standard set of measuring guides, tools used to help the respondent report the volume and dimensions of the food items consumed, were available during interviewing to simplify portion size estimation. The dietary recalls were further characterised as reliable and meeting the minimum criteria by the NCHS if $<25 \%$ of foods were missing descriptive information, $<15 \%$ were missing amounts and the respondent remembered at least one food item per meal. Data considered unreliable were not included in this analysis.

The daily total energy intake for each respondent was quantified and used in the models as a continuous variable. The percentage of daily energy intake obtained from saturated fat, carbohydrates and protein was calculated. Daily intake of dietary fibre in grams was also identified. The International Table of Glycemic Index and Glycemic Load Values 2002 was used in conjunction with the type and amount of food consumed to establish the glycaemic index for the respondent's daily dietary intakes [25]. These values were summed to provide a daily dietary glycaemic index for each respondent.

Other control variables Other control variables included current smoking status, age, sex and BMI. Daily dietary intakes of caffeine in milligrams and number of alcoholic drinks per day were also included. Magnesium intake was included as a categorical variable based on whether respondents met the US Recommended Dietary Allowance for their age and sex [26]. Levels of physical activity were defined by having respondents describe their level of activity over the last 30 days as moderate or vigorous exercise vs neither. These self-assessments were then correlated to the specific daily, leisure-time and sedentary activities the respondent described, and then recoded appropriately. For instance, respondents who described their level of activity as vigorous or moderate, but had not 
Table 1 Demographics for overweight US adults without diagnosed disease (US population estimate, percentage)

*Significant difference $(p<0.05)$ when compared with nonHispanic whites

\begin{tabular}{|c|c|c|c|c|}
\hline & Total & Non-Hispanic white & African-American & Hispanic \\
\hline \multicolumn{5}{|l|}{ All } \\
\hline Sample size & $62,039,724$ & $41,553,945$ & $7,899,451$ & $12,586,328$ \\
\hline Population percentage & 100 & 67.0 & 12.7 & 20.3 \\
\hline \multicolumn{5}{|l|}{ Male } \\
\hline Sample size & $31,899,128$ & $22,617,861$ & $3,179,203$ & $6,102,064$ \\
\hline Population percentage & 100 & 70.9 & 10.0 & 19.1 \\
\hline \multicolumn{5}{|l|}{ Female } \\
\hline Sample size & $30,140,596$ & $18,936,084$ & $4,720,248$ & $6,484,265$ \\
\hline Population percentage & 100 & 62.8 & 15.7 & 21.5 \\
\hline Mean age (SE) & $38.1(0.67)$ & $39.3(0.88)$ & $35.7(0.57)^{*}$ & $35.7(0.93)^{*}$ \\
\hline Mean BMI (SE) & $30.6(0.22)$ & $30.4(0.30)$ & $32.5(0.40)^{*}$ & $30.1(0.27)$ \\
\hline $\begin{array}{l}\text { Dietary recall reliable and } \\
\text { meeting minimum criteria }(\%)\end{array}$ & 95.3 & 95.6 & 92.5 & 96.2 \\
\hline
\end{tabular}

sensitivity. With these models we evaluated the association between insulin sensitivity and ethnicity while controlling for dietary intake as well as other control variables. A $p$ value $<0.05$ was considered significant.

Statistical analysis Because NHANES 1999-2000 was a complex, stratified cluster sample, standard statistical techniques could not be used. Therefore, we used SUDAAN (Research Triangle Institute, Research Triangle, NC, USA), a specialised statistical program that accounts for the complex weighting of the NHANES sample [27]. SUDAAN uses statistical techniques that take into account and correct for unequal probabilities of selection and different response rates, ensuring that the results can be generalised to the non-institutionalised civilian population of the United States. SUDAAN also adjusts the SEs to account for the weighting, stratification and clustering of the complex sampling design, to ensure that expressed $p$ values are valid [28].

Due to the small size and heterogeneity of the 'Other' racial category, this group was not analysed. Means of the dietary intake variables and measures of insulin sensitivity were calculated by ethnicity for individuals with reliable dietary information. ANOVA makes the assumption that every observation has the same variance. This assumption cannot be made due to the sampling design of the NHANES. Thus, we used dummy linear regression as a substitute for ANOVA. In addition to bivariate analyses, linear regressions were performed using fasting insulin as a continuous dependent variable characterising insulin

\section{Results}

The unweighted sample of overweight adults without the conditions to be excluded was 1,562 , which represents over 60 million US adults after appropriate sampling weights are applied. Reliable dietary information was available for $95.3 \%$ of this sample. The demographic characteristics of the population studied are presented in Table 1 .

Table 2 presents the means for dietary intake variables and measures of insulin sensitivity. Dietary differences are seen by ethnicity, with non-Hispanic whites having higher energy, saturated fat and total fat intake, while Hispanics had higher carbohydrate intake and African-Americans had lower fibre intake. Both African-Americans and Hispanics had higher levels of fasting insulin, demonstrating lower insulin sensitivity in comparison with non-Hispanic whites.

Table 3 presents results from linear regressions evaluating insulin sensitivity after controlling for individual dietary variables as well as the other control variables. Being Hispanic and having a higher percentage of energy intake from carbohydrates are associated with lower insulin sen-

Table 2 Means of dietary intakes and insulin sensitivity by ethnicity

\begin{tabular}{lllcc}
\hline & Total & Non-Hispanic white & African-American & Hispanic \\
\hline Total energy intake in kJ (SE) & $9.66(0.17)$ & $10.05(0.24)$ & $8.83(0.27)^{*}$ & $8.87(0.28)^{*}$ \\
Energy intake from protein (SE) & $15.2 \%(0.20)$ & $15.2 \%(0.28)$ & $15.4 \%(0.49)$ & $15.1 \%(0.28)$ \\
Energy intake from carbohydrates $(\mathrm{SE})$ & $50.2 \%(0.51)$ & $49.4 \%(0.61)$ & $51.0 \%(1.10)$ & $52.1 \%(0.79)^{*}$ \\
Energy intake from total fat (SE) & $33.0 \%(0.40)$ & $33.8 \%(0.44)$ & $31.7 \%(0.86)^{*}$ & $31.3 \%(0.64)^{*}$ \\
Energy intake from saturated fat (SE) & $11.1 \%(0.16)$ & $11.5 \%(0.18)$ & $10.3 \%(0.28)^{*}$ & $10.3 \%(0.25)^{*}$ \\
Fibre intake $(\mathrm{g})(\mathrm{SE})$ & $14.6(0.48)$ & $14.9(0.70)$ & $11.1(0.58)^{*}$ & $15.7(0.71)$ \\
Fasting insulin $(\mu \mathrm{U} / \mathrm{ml})(\mathrm{SE})$ & $14.05(0.65)$ & $12.44(0.78)$ & $16.08(1.16)^{*}$ & $16.85(1.83)^{*}$ \\
\hline
\end{tabular}

*Significant difference $(p<0.05)$ when compared with non-Hispanic whites 
Table 3 Adjusted risk of decreased insulin sensitivity by ethnicity and dietary factors ${ }^{\mathrm{a}}$

${ }^{\text {a}}$ Regression also controls for: age, sex, BMI, income, magnesium intake, number of alcoholic drinks per day, caffeine intake, smoking status and exercise

${ }^{\mathrm{b}}$ Referent category for ethnicity dummy variable

\section{Ethnicity}

Non-Hispanic whites ${ }^{b}$

African-American

$-$

Hispanic

$1.41(1.128)$

$-$

$4.67(1.994)$

0.23

Dietary variables

Total energy intake

$0.02(0.001)$

$0.10(0.045)$

$0.18(0.180)$

$0.07(0.121)$

$-0.06(0.033)$
0.03

0.08

0.04

0.33

0.57

0.10 sitivity. As expected, BMI was also associated with lower insulin sensitivity. No other variables were significantly associated with insulin sensitivity.

Table 4 presents results from linear regressions evaluating insulin sensitivity after controlling for the glycaemic index of the dietary intakes and other control variables. Again, being Hispanic and having a higher BMI is associated with lower insulin sensitivity. No other variables were significantly associated with insulin sensitivity.

\section{Discussion}

This study demonstrates that ethnic differences in markers of insulin sensitivity remain even after controlling for dietary differences, suggesting potential inherent metabolic differences between groups or the existence of other cultural differences not reflected in diet or physical activity levels. In fact, although non-Hispanic whites have 'less healthy' diets than Hispanics, they have greater insulin sensitivity. The fact that we found ethnic differences in insulin sensitivity even after accounting for diet reinforces the need to address disparities in diabetes as multifactorial in nature. Thus, while interventions focusing on improving the diets of minority ethnic groups to overcome the health disparity of diabetes are warranted, especially with regard to weight management, other interventions may also be necessary to decrease the prevalence and burden of diabetes.

Table 4 Adjusted risk of decreased insulin sensitivity by ethnicity and daily dietary glycaemic index ${ }^{\mathrm{a}}$

Fasting insulin beta $p$ value
coefficient (SE)

\begin{tabular}{lll}
\hline Ethnicity & & \\
Non-Hispanic whites & & - \\
African-American & - & 0.25 \\
Hispanic & $1.31(1.08)$ & 0.04 \\
Total energy intake & $4.63(2.00)$ & 0.25 \\
Daily dietary glycaemic index & $0.01(0.01)$ & 0.39 \\
\hline
\end{tabular}

${ }^{a}$ Regression also controls for: age, sex, BMI, income, magnesium intake, number of alcoholic drinks per day, caffeine intake, smoking status and exercise

${ }^{\mathrm{b}}$ Referent category for ethnicity dummy variable
The only dietary factor associated with insulin sensitivity, even after adjustment for BMI and ethnicity, is the percentage of total daily energy intake from carbohydrates. Having a lower percentage of energy intake from carbohydrates is associated with higher insulin sensitivity. These results suggest that the effects of low carbohydrate diets should be studied in diabetic patients and those at risk of developing diabetes, since these diets may confer specific benefits to this population by increasing insulin sensitivity.

There are limitations to this study. First, the measures of diet were based on a 24-h dietary history, and it is possible that individuals could change their diets over time. However, studies have shown that middle-aged people are likely to have a stable nutrient intake over many years [28, 29]. Furthermore, studies assessing the validity of 24-h recalls demonstrate adequate accuracy for epidemiological studies [30-33]. Second, it is possible that it is not carbohydrates themselves, but a nutrient linked to carbohydrate intake that leads to the associations seen in this study. Further research is required to assess this question, as this study focuses on macronutrient intake.

In conclusion, the differences in dietary intake seen in different ethnic groups do not completely account for the disparities in insulin sensitivity. Further study is needed to define the inherent ethnic metabolic factors, as well as other non-dietary factors, that affect insulin sensitivity. This may help in the development of novel interventions.

Acknowledgements This study was funded in part through grants 1 D14 HP 00161-02 and 2 D12 HP-00023-04 from Health Resources and Services Administration and grant 1 P30 AG 021677 from South Carolina Resource Centers for Minority Aging Research.

\section{References}

1. America Diabetes Association (2002) Screening for diabetes. Diabetes Care 25:S21-S24

2. Mokdad AH, Ford ES, Bowman BA et al (2003) Prevalence of obesity, diabetes, and obesity-related health risk factors, 2001. JAMA 289:76-79

3. Rubin RJ, Altman WM, Mendelson DN (1994) Health care expenditures for people with diabetes mellitus. J Clin Endocrinol Metab 78:809A-809F 
4. Eastman RC, Javitt JC, Herman WH et al (1997) Model of complications of NIDDM. Analysis of the health benefits and cost-effectiveness of treating NIDDM with the goal of normoglycemia. Diabetes Care 20:735-744

5. Wilson PW (1998) Diabetes mellitus and coronary heart disease. Am J Kidney Dis 32(5 Suppl 3):S89-S100

6. Sanchez-Thorin JC (1998) The epidemiology of diabetes mellitus and diabetic retinopathy. Int Ophthalmol Clin 38:1118

7. Karter AJ, Assiamira Ferrara A, Liu JY, Moffet HH, Ackerson LM, Selby JV (2002) Ethnic disparities in diabetic complications in an insured population. JAMA 287:2519-2527

8. Kingston RS, Smith JP (1997) Socioeconomic status and racial and ethnic differences in functional status associated with chronic diseases. Am J Public Health 87:805-810

9. MMWR (1999) Self-reported prevalence of diabetes among Hispanics-United States, 1994-1997. Mor Mortal Wkly Rep 48:8-12

10. American Diabetes Association (2000) Nutrition recommendations and principles for people with diabetes mellitus. Diabetes Care 23(Suppl 1):S43-S46

11. Riccardi G, Rivellese AA (2000) Dietary treatment of the metabolic syndrome - the optimal diet. Br J Nutr 1:S143-S148

12. Kwan LL, Bermudez OI, Tucker KL (2002) Low vitamin B-12 intake and status are more prevalent in Hispanic older adults of Caribbean origin than in neighborhood-matched non-Hispanic whites. J Nutr 132:2059-2064

13. Gans KM, Burkholder GJ, Risica PM, Lasater TM (2003) Baseline fat-related dietary behaviors of white, Hispanic, and black participants in a cholesterol screening and education project in New England. J Am Diet Assoc 103:699-706

14. Kolonel LN, Henderson BE, Hankin JH et al (2000) A multiethnic cohort in Hawaii and Los Angeles: baseline characteristics. Am J Epidemiol 151:346-357

15. Robinson ME, Hunter PH (2001) Nutritional assessment of a predominantly African-American inner-city clinic population. WMJ 100:32-38

16. Kronsberg SS, Obarzanek E, Affenito SG et al (2003) Macronutrient intake of black and white adolescent girls over 10 years: the NHLBI Growth and Health Study. J Am Diet Assoc 103:852-860

17. Lovejoy JC, Champagne CM, Smith SR, de Jonge L, Xie H (2001) Ethnic differences in dietary intakes, physical activity, and energy expenditure in middle-aged, premenopausal women: the Healthy Transitions Study. Am J Clin Nutr 74:90-95

18. Ford ES, Mokdad AH (2003) Dietary magnesium intake in a national sample of US adults. J Nutr 133:2879-2882

19. Arab L, Carriquiry A, Steck-Scott S, Gaudet MM (2003) Ethnic differences in the nutrient intake adequacy of premenopausal US women: results from the Third National Health Examination Survey. J Am Diet Assoc 103:1008-1014
20. Haffner SM, D’Agostino R Jr, Saad MF et al (1996) Increased insulin resistance and insulin secretion in nondiabetic African Americans and Hispanics compared with non-Hispanic whites: the Insulin Resistance Atherosclerosis Study. Diabetes 45:742748

21. Haffner SM, Bowsher RR, Mykkanen L et al (1994) Proinsulin and specific insulin concentration in high and low risk populations for non-insulin dependent diabetes mellitus. Diabetes 43:1490-1493

22. Clinical guidelines on the identification, evaluation and treatment of overweight and obesity in adults: the evidence report (1998). NIH publication, No. 98-4083. Sept 1998. National Institutes of Health. National Heart, Lung, and Blood Institute in cooperation with the National Institute of Diabetes and Digestive and Kidney Diseases

23. McAuley KA, Williams SM, Mann JI et al (2001) Diagnosing insulin resistance in the general population. Diabetes Care $24: 460-464$

24. Laakso M (1993) How good a marker is insulin level for insulin resistance? Am J Epidemiol 137:959-965

25. Foster-Powell K, Holt SHA, Brand-Miller JC (2002) International table of glycemic index and glycemic load values: 2002. Am J Clin Nutr 76:5-56

26. The US Office of Dietary Supplements of the National Intitutes of Health. Available from http://www.ods.od.nih.gov/factsheets/ cc/magn.html, accessed 1 December 2005

27. Vuguin P, Saenger P, Dimartino-Nardi J (2001) Fasting glucose insulin ratio: a useful measure of insulin resistance in girls with premature adrenarche. J Clin Endocrinol Metab 86:4618-4621

28. Jensen OM, Whrendorf J, Rosenquist A, Geser A (1984) The reliability of questionnaire-derived historical dietary information and temporal stability of food habits in individuals. Am J Epidemiol 120:281-290

29. James GD, Sealey JE, Alderman MH, Laragh JH (1993) Year to year stability of urine sodium, potassium, aldosterone and PRA in normotensive men and women. Am J Hypertens 6:86A-90A

30. Conway JM, Ingwersen LA, Moshfegh AJ (2004) Accuracy of dietary recall using the SDA five-step multiple-pass method in men: an observational validation study. J Am Diet Assoc 104:595-603

31. Sharma M, Rao M, Jacob S, Jacob CK (1998) Validation of 24-hour dietary recall: a study in hemodialysis patients. J Renal Nutr 8:199-202

32. Buzzard IM, Faucett CL, Jeffery RW et al (1996) Monitoring dietary change in a low-fat diet intervention study: advantages of using 24-hour dietary recalls vs food records. J Am Diet Assoc 96:574-579

33. Karvetti RL, Knuts LR (1985) Validity of the 24-hour dietary recall. J Am Diet Assoc 85:1437-1442 